\title{
Implementación de una metodología para la propagación y crecimiento del romerillo
}

Implementación de una metodología para la propagación y crecimiento del romerillo

Jorge Eduardo Herrera Cabezas. ${ }^{1} \&$ Yasser Alejandro Chim Chi. ${ }^{2}$

\begin{abstract}
.
DOI: https://doi.org/10.33262/concienciadigital.v4i2.1677

Romerillo (Podocarpus oleifolius) is an autochthonous forest conifer of Ecuador that can be considered vulnerable. It is among the most important species as a sustainable and renewable source in the production of wooden articles, and as an element in the reforestation and controlled regeneration of natural forests. The present investigation proposes a methodology for the propagation of romerillo based on the influence of different organic substrates (humus, peat and manure) and altitude floors: Alao community, San Andrés parish and Juan de Velasco parish, belonging to the province of Chimborazo; Through taking horizontal and vertical growth data in a period of nine months, the incidence on growth was determined, which resulted in that the altitudinal floor does not have a significant difference in the growth of the plant; on the contrary, the use of different organic substrates affect its growth. When using smoke, there was $78 \%$ survival, with a growth rate of $3.01 \mathrm{~cm}$ per month and an average vertical growth of 24.03 $\mathrm{cm}$. The use of organic substrates is necessary to increase the probability of propagation and adaptation of the species, regardless of the altitude floor. With these results, the methodology can be applied on a large scale in order to preserve vulnerable or threatened species.
\end{abstract}

\footnotetext{
${ }^{1}$ Universidad Católica de Cuenca, jehc300386@gmail.com, https://orcid.org/0000-0001-7130-4704

2 Tecnológico Nacional de México, yachim@itescam.edu.mx, https://orcid.org/0000-0002-4720-8445
} 
Keywords: Podocarpus oleifolius, propagation, organic substrates, altitudinal floors, methodology.

\section{Resumen.}

Introducción.- El romerillo (Podocarpus oleifolius) es una conífera forestal vernácula del Ecuador que puede reflexionar como asequible. Se encuentra entre las especies de mayor importancia como fuente sustentable y renovable en la producción de artículos de madera, y como elemento en la reforestación y regeneración controlada de bosques naturales. La presente investigación propone una metodología para la propagación del romerillo sustentado en la influencia de los diferentes sustratos orgánicos (humus, turba y estiércol) y pisos altitudinales: comunidad de Alao, parroquia San Andrés y la parroquia Juan de Velasco, pertenecientes a la provincia de Chimborazo; a través de toma de datos de crecimiento horizontal y vertical en un período de nueve meses, se determinó la incidencia en el crecimiento el cual dio como resultado que el piso altitudinal no tiene una diferencia significativa en el crecimiento de la planta; por el contrario, el uso de diferentes sustratos orgánicos inciden en su crecimiento. Al usar humos existió el 78\% de supervivencia, con una tasa de crecimiento de $3,01 \mathrm{~cm}$ por mes y un promedio de crecimiento vertical de $24,03 \mathrm{~cm}$. El uso de sustratos orgánicos es necesario para aumentar la probabilidad de propagación y adaptación de la especie, indiferentemente del piso altitudinal. Con estos resultados se puede aplicar la metodología a gran escala a fin de preservar especies vulnerables o amenazadas.

Palabras claves: Podocarpus oleifolius, propagación, sustratos orgánicos, pisos altitudinales, metodología.

\section{Introducción.}

Los bosques son origen de recursos disponibles por el ser humanitario; el alto grado de mediación de acciones antropogénicas, el uso no llevadero de recursos naturales, la deforestación, la ignominia de las escenarios de los entornos imprescindible la confección de saberes que busquen la manutención de la biodiversidad, lo cual es uno de los antesesores jactancias de la humanidad. En América Latina se descubren alrededor del $25 \%$ de los bosques del mundo y cerca del $50 \%$ de selvas cálidas del asteroide, es un terruño que alberga una alta biodiversidad. Sin embargo, es una de las comarcas con un repertorio per cápita de deforestación más grande de la creación (Keipi, 2000). De acuerdo a Global Forest Watch (2020) entre el 2002 y el 2019 fueron deforestados un total de 60,5 millones de hectáreas de bosque húmedo primario a nivel cosmopolita, lo que representa el $16 \%$ de la pérdida total de abrigo forestal.

En el Ecuador se rastreó una pérdida de 173 mil hectáreas de bosque húmedo primario a causa de deforestación, entre el 2002 y 2019, lo que significa el 22\% del detrimento total 
de cobertura arbórea; las provincias de Sucumbíos, Esmeraldas, Orellana y Manabí son las más presuntuosas por la deforestación y engloban el 54\% de la deforestación del país (Global Forest Watch, 2020). En este contrariado. Reinos de árboles como el romerillo (Podocarpus oleifolius) conciernen a la caterva de especies que pueden exteriorizar un grado de inminencia debido a que su trascendental uso es para la manufactura de muebles, por lo que se ha ocasionado una tala imperceptible de la especie sin reforestación de la mismo. La ambigua se incremente, debido a que la variedad exterioriza un valor ecológico agregado al permitir la mantenimiento de los páramos y cuencas hidrográficas tanto en la provincia de Chimborazo como en el resto del país. Es incuestionable que la nebulosa de la deforestación, exige operaciones inmediatas por parte de las autoridades; una disyuntiva que ha tomado el Estado Ecuatoriano ha sido el perfeccionamiento de programas y proyectos encaminados en la depreciación de la deforestación y la conservación de los bosques en el país, como lo es el Programa de Restauración Forestal del Ministerio del Ambiente y Agua del Ecuador (MAEE).

La desventaja de responsabilidad vegetal puede ocasionar el surgimiento de riesgos ambientales tales como inclinaciones de tierras, pérdida de biodiversidad, transformaciones en el clima, pérdida de nacimientos hídricas, entre otros. Por lo que es inexcusable desarrollar una metodología para la trasmisión de la especie de una manera verosímil, para la cual, se experimentará las circunstancias de supervivencia y crecimiento de la especie en diferentes pisos altitudinales y con diferentes sustratos, con ello se podrá comprobar las mejores condiciones posibles para la propagación de la especie y de esta manera recuperar áreas deforestadas. Cabe indicar que los bosques de Podocarpus pueden ser utilizados con otros fines para la generación de recursos económicos, como atractivo turístico, a través del fortalecimiento de programas de turismo comunitario, estos árboles pueden llegar a tener hasta 6 o 7 metros de diámetro y hasta 40 metros de altura, por lo que podrían constituirse como un atractivo turístico.

La reparación del suelo no se formaliza de manera técnica y con las ilustraciones pasaderas sobre los requisitos que tiene la variedad a reforestar superficies repudiadas; sobre todo, existe muy poca indagación sobre sustratos que exterioricen condiciones imponderables para la manufactura de las especies forestales en peligro de decadencia. Por lo que, se debe perpetrar una justiprecio que incluya la combinación de la vegetación, la composición de las especies y la cobertura del suelo antes de iniciar la recuperación para especies endémicas (Moura, y otros, 2016). Por tanto, para bosquejar un programa de reforestación triunfante, unos de los coeficientes principales es elegir el sustrato correcto, el cual influye directamente en la germinación y en el perfeccionamiento de las plántulas, y en la generación de supervivencia de los especímenes plantados. Esto, hace inexcusable evaluar las propiedades que favorezcan el crecimiento.

\section{Metodologia.}

Para el vigente proyecto se aplicará la investigación exploratoria, la cual establece Díaz y Núñez (2016), la cual se basa en la descripción de un fenómeno nuevo o con 
información escasa; por lo que, la investigación tiene como finalidad de identificar, reconocer y familiarizarse con los objetos de estudio.

Para la fase experimental se consideraron 900 individuos obtenidos del vivero forestal ubicado en la comunidad de Alao; las plántulas consideradas, al alcanzar una altura de entre 18 a 20 centímetros en aproximadamente 9 meses, fueron trasplantadas en los sitios designados. Adicionalmente, se observó que las plántulas posean características que favorezcan su supervivencia como: ramificación, tallo y raíces.

El proceso experimental duró 9 meses, en los cuales fueron plantadas 100 individuos por cada piso altitudinal y por cada sustrato seleccionado, danto el total de 900 individuos plantados, dividas en tres bloques.

Cabe indicar que la recolección de información en cuanto se refiere a la variación de crecimiento vertical (altura) y el conteo de individuos vivos se tomó mediciones mensuales; mientras que la variación del crecimiento horizontal (diámetro) se realizó cada tres meses. La información fue recolectada cada mes iniciando en octubre de 2018 y finalizando en junio de 2019, llevando un registro de campo, en donde se registró la altura de la plántula, diámetro del tallo y el conteo de individuos vivos y muertos.

Los sitios seleccionados donde se trasplantó a los individuos corresponden a tres pisos altitudinales, designados con los diferentes sustratos que permitió desarrollar los conocimientos científicos relacionados con la propagación de la especie romerillo (Podocarpus oleifolius). Respeto a los sustratos seleccionados para el crecimiento y desarrollo de los individuos fueron: estiércol de ganado, humus y turba.

Los sitios seleccionados presentan características de suelo, condiciones de altitud, humedad y temperatura que favorecen el desarrollo y propagación de la especie:

- PA - Piso A / Alao; Montano; 3.250 metros sobre el nivel del mar; Provincia: Chimborazo, Cantón: Chambo, Comunidad: Alao.

- PB- Piso B / Juan de Velasco; Alpino; Más de 4.000 metros sobre el nivel del mar; Provincia: Chimborazo, Cantón: Colta, Parroquia: Juan de Velasco.

- PC- Piso C / Tuntatacto; Subalpino; 3.280 - 3.600 metros sobre el nivel del mar; Provincia: Chimborazo, Cantón: Guano, Comunidad: Tuntatacto

Los tratamientos del experimento se constituyeron por la aplicación de los tres sustratos, que fueron utilizados en los tres pisos altitudinales, cada uno de los sustratos fue aplicado en la misma cantidad, a un grupo determinado de plantas, lo cual se presenta en la Tabla 1. 
Vol. 4, N², p. 277-291, abril-junio, 2021

Tabla 1.- Tratamientos con diferentes sustratos

\begin{tabular}{|c|c|c|c|c|}
\hline Trat. & PA & Sust. & Cód. & Descripción \\
\hline \multirow{3}{*}{ T1 } & A & 1 & $\mathrm{~A} 1$ & $\begin{array}{l}\text { Piso Montano, sitio Alao con sustrato de Estiércol } \\
\text { de ganado }\end{array}$ \\
\hline & $A$ & 2 & $\mathrm{~A} 2$ & Piso Montano, sitio Alao con sustrato de Humus \\
\hline & $A$ & 3 & A3 & Piso Montano, sitio Alao con sustrato de Turba \\
\hline \multirow{3}{*}{ T2 } & B & 1 & B1 & $\begin{array}{l}\text { Piso Alpino, sitio Juan de Velasco con sustrato de } \\
\text { Estiércol de ganado }\end{array}$ \\
\hline & B & 2 & B2 & $\begin{array}{c}\text { Piso Alpino, sitio Juan de Velasco con sustrato de } \\
\text { Humus }\end{array}$ \\
\hline & B & 3 & B3 & $\begin{array}{c}\text { Piso Alpino, sitio Juan de Velasco con sustrato de } \\
\text { Turba }\end{array}$ \\
\hline \multirow{3}{*}{ T3 } & $\mathrm{C}$ & 1 & $\mathrm{C} 1$ & $\begin{array}{l}\text { Piso Subalpino, sitio Tuntatacto con sustrato de } \\
\text { Estiércol de ganado }\end{array}$ \\
\hline & $\mathrm{C}$ & 2 & $\mathrm{C} 2$ & $\begin{array}{l}\text { Piso Subalpino, sitio Tuntatacto con sustrato de } \\
\text { Humus }\end{array}$ \\
\hline & $\mathrm{C}$ & 3 & C3 & $\begin{array}{c}\text { Piso Subalpino, sitio Tuntatacto con sustrato de } \\
\text { Turba }\end{array}$ \\
\hline
\end{tabular}

Trat. $=$ Tratamiento; PA = Piso Altitudinal; Sust. = Sustrato; Cód. $=$ Código.

Fuente: Elaboración propia.

Previo al trasplante, fue conveniente "rozar" el espacio en el cual fueron colocadas las plantas; esto quiere decir que fue necesario despejar el terreno o desbrozar la vegetación que pudo crear competencia al árbol en su entorno inmediato. Una vez rozado se el suelo fue preparado para facilitar el arraigo y la primera etapa de desarrollo de la planta por lo que se realizó un hoyo de plantación de dimensiones aproximadas de 30 x 30 x $30 \mathrm{~cm}$. En el lugar que fue trasplantado, se realizó una validación periódicamente y toma de información.

Cada una de las 900 plántulas fue ubicada de acuerdo a los sitios y sustratos seleccionados en los sitios definidos, los cuales fueron laderas de los ríos o quebradas y alrededor de los terrenos como cortinas rompe vientos y pajonales. La distancia entre hoyos varió según los siguientes elementos: la densidad de los árboles por hectárea, clima, especies utilizadas, suelos, topografía y uso final de las plántulas; tomando en cuenta lo descrito, se tomó como distancia de siembre entre plántula $3 \mathrm{~m}$ x 3m. 


\section{Resultados.}

\section{Crecimiento Vertical}

Los resultados del crecimiento vertical se indican en la Tabla 2. Respecto al tratamiento T1, el código A1, se registró valores de crecimiento promedio de $23,83 \mathrm{~cm}$, lo que representa un crecimiento $2,99 \mathrm{~cm}$ cada mes. Se tuvo un valor inicial promedio de 9,23 cm y la planta creció en promedio hasta una altura de 33,11 cm. En el código A2, se registró valores de crecimiento promedio de $23,83 \mathrm{~cm}$, lo que representa un crecimiento $2,98 \mathrm{~cm}$ cada mes. Se tuvo un valor inicial promedio de $9,24 \mathrm{~cm}$ y la planta creció en promedio hasta una altura de 33,90 cm. En referencia al código A3, se registró valores de crecimiento promedio de $24,66 \mathrm{~cm}$, lo que representa un crecimiento $3,08 \mathrm{~cm}$ cada mes. Se tuvo un valor inicial promedio de $9,30 \mathrm{~cm}$ y la planta creció en promedio hasta una altura de $33,90 \mathrm{~cm}$.

Para el tratamiento T2, en el código B1, se registró valores de crecimiento promedio de $24,03 \mathrm{~cm}$, lo que representa un crecimiento $2,95 \mathrm{~cm}$ cada mes. Se tuvo un valor inicial promedio de 9,19 cm y la planta creció en promedio hasta una altura de 33,22 cm. Respecto al código B2, se registró valores de crecimiento promedio de $24,26 \mathrm{~cm}$, lo que representa un crecimiento $3,05 \mathrm{~cm}$ cada mes. Se tuvo un valor inicial promedio de 9,25 $\mathrm{cm}$ y la planta creció en promedio hasta una altura de $33,27 \mathrm{~cm}$. En referencia al código B3, se registró valores de crecimiento promedio de $23,99 \mathrm{~cm}$, lo que representa un crecimiento 3,01 cm cada mes. Se tuvo un valor inicial promedio de 9,24 cm y la planta creció en promedio hasta una altura de $33,23 \mathrm{~cm}$.

Sobre el tratamiento T3, en el código C1, se registró valores de crecimiento promedio de $26,18 \mathrm{~cm}$, lo que representa un crecimiento $3,27 \mathrm{~cm}$ cada mes. Se tuvo un valor inicial promedio de 9,24 cm y la planta creció en promedio hasta una altura de $33,23 \mathrm{~cm}$. Respecto al código C2, se registró valores de crecimiento promedio de $24,04 \mathrm{~cm}$, lo que representa un crecimiento $3,03 \mathrm{~cm}$ cada mes. Se tuvo un valor inicial promedio de 9,24 cm y la planta creció en promedio hasta una altura de 33,28 cm. En referencia al código C3, se registró valores de crecimiento promedio de $24,34 \mathrm{~cm}$, lo que representa un crecimiento 3,04 cm cada mes. Se tuvo un valor inicial promedio de 9,23 cm y la planta creció en promedio hasta una altura de $33,30 \mathrm{~cm}$.

Tabla 2.- Resumen sobre crecimiento vertical

\begin{tabular}{cccccccc}
\hline PA & $\mathbf{S}$ & $\mathbf{H}_{\mathbf{o}}$ & $\mathbf{H}_{\mathbf{f}}$ & $\mathbf{P C}$ & $\mathbf{P C M}$ & $\mathbf{S F}$ & $\mathbf{M F}$ \\
\hline & 1 & 9,23 & 33,11 & 23,89 & 2,99 & 59 & 41 \\
$\mathbf{A}$ & 2 & 9,24 & 33,90 & 23,83 & 2,98 & 79 & 21 \\
& 3 & 9,30 & 33,90 & 24,40 & 3,05 & 71 & 29 \\
\hline
\end{tabular}


Vol. 4, N², p. 277-291, abril-junio, 2021

\begin{tabular}{|c|c|c|c|c|c|c|c|}
\hline PA & $\mathbf{S}$ & $\mathbf{H}_{\mathrm{o}}$ & $\mathbf{H}_{\mathrm{f}}$ & PC & PCM & SF & MF \\
\hline & 1 & 9,19 & 33,22 & 24,03 & 2,95 & 56 & 44 \\
\hline \multirow[t]{3}{*}{ B } & 2 & 9,25 & 33,27 & 24,02 & 3,03 & 76 & 24 \\
\hline & 3 & 9,24 & 33,23 & 23,99 & 3,01 & 70 & 30 \\
\hline & 1 & 9,23 & 33,23 & 24,00 & 3,00 & 59 & 41 \\
\hline \multirow[t]{2}{*}{ C } & 2 & 9,24 & 33,28 & 24,04 & 3,03 & 80 & 20 \\
\hline & 3 & 9,23 & 33,30 & 24,07 & 3,01 & 71 & 29 \\
\hline
\end{tabular}

PA = Piso Altitudinal; $\mathrm{S}=$ Sustrato; $\mathrm{H}_{\mathrm{o}}=$ medición inicial de altura de la planta $(\mathrm{cm}) ; \mathrm{H}_{\mathrm{o}}=$ medición final de altura de la planta $(\mathrm{cm}) ; \mathrm{PC}=$ promedio de crecimiento $(\mathrm{cm}) ; \mathrm{PCM}=$ Promedio crecimiento mensual; $\mathrm{SF}=$ cantidad de individuos sobrevivientes en el conteo final; $\mathrm{MF}=$ cantidad de individuos muertos en el conteo final. Fuente: Elaboración propia.

\section{Crecimiento Horizontal}

Los resultados del crecimiento horizontal se indican en la Tabla 3. Respecto al tratamiento T1, el código A1, se registró valores de crecimiento promedio de $27,19 \mathrm{~mm}$, lo que representa un crecimiento 3,40 mm cada mes. Se tuvo un valor inicial promedio de 3,96 mm y la planta creció en promedio hasta un ancho de 31,15 mm. En el código A2, se registró valores de crecimiento promedio de 27,64 mm, lo que representa un crecimiento $3,45 \mathrm{~mm}$ cada mes. Se tuvo un valor inicial promedio de 4,01 $\mathrm{mm}$ y la planta creció en promedio hasta un ancho de 31,65 mm. En referencia al código A3, se registró valores de crecimiento promedio de $27,79 \mathrm{~mm}$, lo que representa un crecimiento $3,47 \mathrm{~mm}$ cada mes. Se tuvo un valor inicial promedio de 3,68 $\mathrm{mm}$ y la planta creció en promedio hasta un ancho de $31,46 \mathrm{~mm}$.

Para el tratamiento T2, en el código B1, se registró valores de crecimiento promedio de $27,48 \mathrm{~mm}$, lo que representa un crecimiento $3,44 \mathrm{~mm}$ cada mes. Se tuvo un valor inicial promedio de 3,74 mm y la planta creció en promedio hasta un ancho de 31,22 mm. Respecto al código B2, se registró valores de crecimiento promedio de 25,29 mm, lo que representa un crecimiento 3,16 mm cada mes. Se tuvo un valor inicial promedio de 4,01 $\mathrm{mm}$ y la planta creció en promedio hasta un ancho de 31,65 mm. En referencia al código B3, se registró valores de crecimiento promedio de $27,95 \mathrm{~mm}$, lo que representa un crecimiento 3,49 mm cada mes. Se tuvo un valor inicial promedio de $3,76 \mathrm{~mm}$ y la planta creció en promedio hasta un ancho de $31,71 \mathrm{~mm}$.

Sobre el tratamiento T3, en el código C1, se registró valores de crecimiento promedio de $27,82 \mathrm{~mm}$, lo que representa un crecimiento 3,67 mm cada mes. Se tuvo un valor inicial promedio de 3,75 mm y la planta creció en promedio hasta un ancho de 33,08 mm. Respecto al código C2, se registró valores de crecimiento promedio de 27,80 mm, lo que representa un crecimiento $3,48 \mathrm{~mm}$ cada mes. Se tuvo un valor inicial promedio de 4,01 
mm y la planta creció en promedio hasta un ancho de 31,65 mm. En referencia al código C3, se registró valores de crecimiento promedio de $27,77 \mathrm{~mm}$, lo que representa un crecimiento $3,47 \mathrm{~mm}$ cada mes. Se tuvo un valor inicial promedio de $3,84 \mathrm{~mm}$ y la planta creció en promedio hasta un ancho de $31,61 \mathrm{~mm}$.

Tabla 3.- Resumen sobre crecimiento horizontal

\begin{tabular}{|c|c|c|c|c|c|c|}
\hline \multirow[b]{2}{*}{ Mes } & \multicolumn{2}{|c|}{ A1 } & \multicolumn{2}{|c|}{ A2 } & \multicolumn{2}{|c|}{ A3 } \\
\hline & $\begin{array}{l}\text { Promedio } \\
\text { (cm) }\end{array}$ & $\begin{array}{c}\text { No. } \\
\text { Individuos }\end{array}$ & $\begin{array}{l}\text { Promedio } \\
\text { (cm) }\end{array}$ & $\begin{array}{c}\text { No. } \\
\text { Individuos }\end{array}$ & $\begin{array}{l}\text { Promedio } \\
\text { (cm) }\end{array}$ & $\begin{array}{c}\text { No. } \\
\text { Individuos }\end{array}$ \\
\hline Dic-18 & $3,96 \pm 0,12$ & 59 & $4,01 \pm 0,14$ & 79 & $3,68 \pm 0,69$ & 79 \\
\hline Mar-19 & $21,43 \pm 0,09$ & 59 & $22,44 \pm 0,14$ & 79 & $21,82 \pm 0,14$ & 79 \\
\hline \multirow[t]{2}{*}{ Jun-19 } & $31,15 \pm 0,09$ & 59 & $31,65 \pm 0,14$ & 79 & $31,46 \pm 0,14$ & 79 \\
\hline & \multicolumn{2}{|c|}{ B1 } & \multicolumn{2}{|c|}{ B2 } & \multicolumn{2}{|c|}{ B3 } \\
\hline Dic-18 & $3,74 \pm 0,46$ & 71 & $3,80 \pm 0,28$ & 59 & $3,76 \pm 0,45$ & 79 \\
\hline Mar-19 & $21,66 \pm 0,32$ & 71 & $21,30 \pm 0,29$ & 59 & $21,17 \pm 0,28$ & 79 \\
\hline \multirow[t]{2}{*}{ Jun-19 } & $31,22 \pm 0,42$ & 71 & $29,09 \pm 0,11$ & 56 & $31,71 \pm 0,28$ & 76 \\
\hline & \multicolumn{2}{|c|}{ C1 } & \multicolumn{2}{|c|}{$\mathrm{C} 2$} & \multicolumn{2}{|c|}{ C3 } \\
\hline Dic-18 & $3,75 \pm 0,15$ & 59 & $3,89 \pm 0,27$ & 80 & $3,84 \pm 0,63$ & 71 \\
\hline Mar-19 & $31,64 \pm 0,17$ & 59 & $20,78 \pm 0,29$ & 80 & $24,91 \pm 0,27$ & 71 \\
\hline Jun-19 & $33,08 \pm 0,18$ & 59 & $31,71 \pm 0,29$ & 80 & $31,61 \pm 0,38$ & 71 \\
\hline
\end{tabular}

Fuente: Elaboración propia.

\section{Supervivencia}

Respecto al porcentaje de supervivencia de cada 100 individuos plantadas en cada código (ver Figura 1), se tiene que para el tratamiento T1, el código A1, se registró el 91\% de individuos vivos y $9 \%$ de muertos en el primer mes de experimento; durante el segundo mes del experimento el porcentaje de muertas aumentó a $41 \%$, dejando el $59 \%$ de individuos vivos. Para el código A2, se obtuvo, durante el primer mes, un $82 \%$ de individuos vivos y $18 \%$ de muertos; durante el segundo mes del experimento el porcentaje de plantas muertas aumentó a 21\%, dejando el $79 \%$ de individuos vivos. En los códigos A1 y A2, el porcentaje no varió a partir del segundo mes. Para el código A3 se registró el 
$71 \%$ de individuos vivos y $29 \%$ de muertos; porcentaje que no varió hasta el final de la toma de datos.

Respecto al Tratamiento T2, el código B1 presentó 59\% de individuos vivos y $41 \%$ de mortalidad hasta el séptimo mes de experimento, en el cual el porcentaje de muertas aumentó a 44\%, dejando el 56\% de individuos vivos; a partir del séptimo mes se encontró una estabilidad de las plántulas y el porcentaje no varió hasta el final de la toma de datos. Para el código B2 se registró $91 \%$ de individuos vivos y $9 \%$ de muertos, hasta el cuarto mes, en el cual el porcentaje de muertas aumentó a 20\%, dejando el $80 \%$ de individuos vivos; durante el quinto mes el porcentaje de sobrevivientes disminuyó a 79\%; durante el séptimo mes el valor continuó disminuyendo a $78 \%$, en el mes siguiente se situó en $76 \%$ y para el final de la toma de datos el valor se registró en $75 \%$ de sobrevivientes. Para el código B3 se registraron $71 \%$ de individuos vivos y $29 \%$ de muertos; porcentaje que varió durante el octavo mes, teniendo un porcentaje de sobrevivientes del $70 \%$, lo que se mantuvo hasta el final de la toma de datos.

Respecto al Tratamiento T3, el código C1 durante el primer mes se registró $77 \%$ de individuos vivos y $23 \%$ de muertos; por lo contrario, durante el segundo mes el porcentaje de muertas aumentó a $41 \%$, dejando el $59 \%$ de individuos vivos. Para el código C2 durante el primer mes registró $83 \%$ de individuos vivos y $17 \%$ de muertos; durante el segundo mes del experimento el porcentaje de muertas aumentó a 20\%, dejando el $80 \%$ de individuos vivos. Tanto para el código $\mathrm{C} 1$ y C2, a partir del segundo mes se encontró una estabilidad de las plántulas y el porcentaje no vario hasta el final de la toma de datos. Para el código C3 durante el primer mes se registraron 71\% de individuos vivos y 29\% de mortalidad; porcentaje que no varió hasta el final de la toma de datos.

\section{Análisis estadístico}

Para el análisis estadístico se realizó un análisis de varianza de dos factores, considerando que los dos factores serían: el piso altitudinal y el tipo de sustrato, cada uno con tres niveles de efectos fijos. Los niveles se cruzan formando 9 condiciones experimentales distintas.

Realizando un análisis univariado de variación a fin de obtener una prueba de los efectos inter-sujetos, considerando como variable dependiente el crecimiento total de las especies, es decir la cantidad de altura total que se obtuvo con la medición final restándole la medición inicial de altura. Se obtuvo los resultados indicados en la tabla 4; de acuerdo a la prueba se puede verificar según a los niveles de significancia que la interacción del Sustrato tiene un p-valor menor a 0,05 por lo que se rechaza la hipótesis nula que indica que las variaciones de crecimiento debido al sustrato son iguales y no tienen relación con el sustrato aplicado. Por el contrario, el p-valor del piso altitudinal se encuentra en 0,725 lo que indicaría que no influye el piso altitudinal en el crecimiento de las plantas. Así mismo, la interacción entre el Piso Altitudinal y los diferentes sustratos no tienen una diferencia significativa, lo que indica que no existe una relación estadística que pueda 
demostrar una interacción entre los sustratos y el piso altitudinal. El piso altitudinal no influye en el crecimiento de la planta; sin embargo, los diferentes sustratos empleados influyen en el crecimiento de la planta.

Tabla 4.- Prueba de efectos inter-sujetos. Modelo general lineal, multivariante.

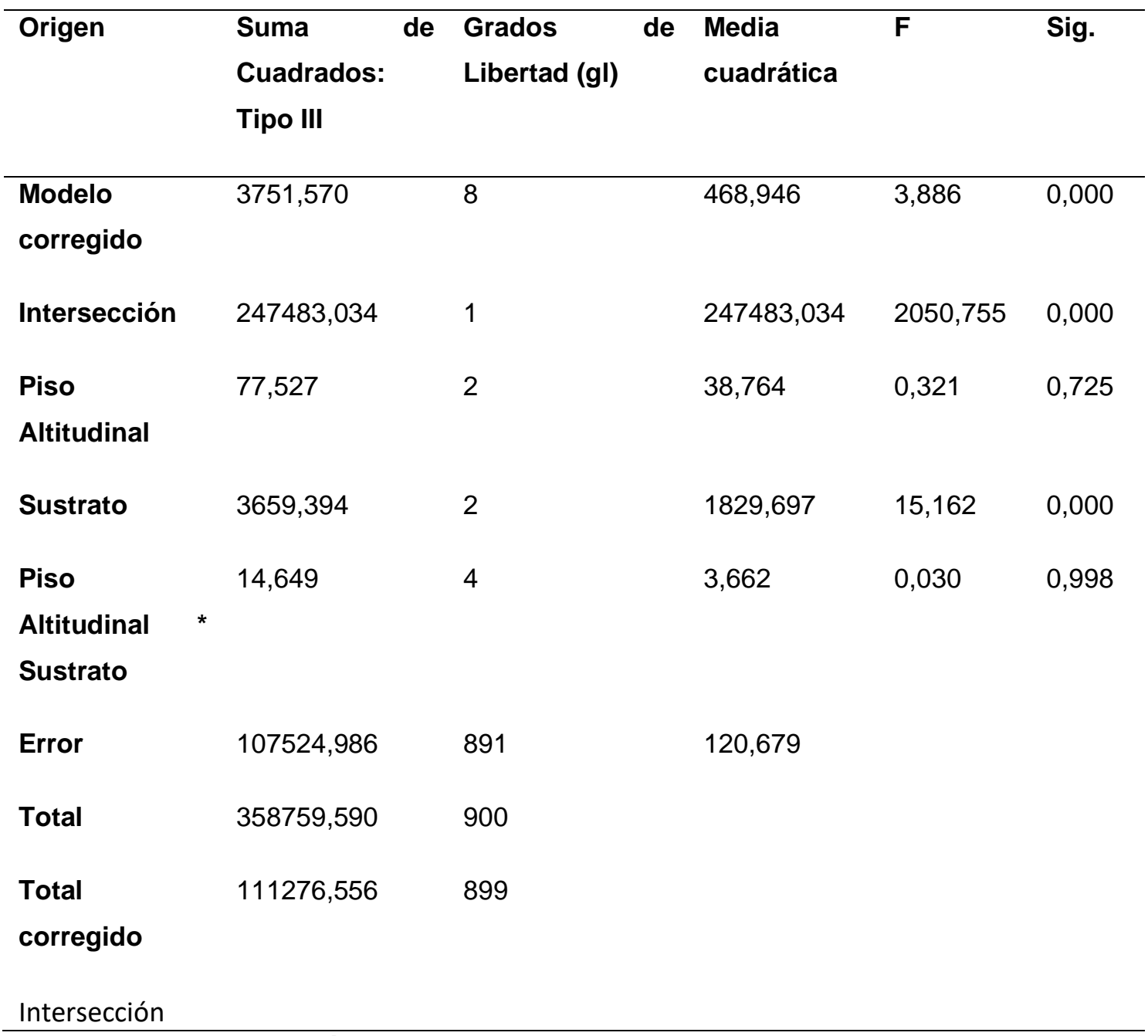

Fuente: Elaboración propia.

$\mathrm{Al}$ realizar pruebas multivariantes (Tabla 5) considerando los factores de piso altitudinal, el sustrato, sus interacciones y las 9 mediciones realizadas a lo largo del experimento (Crecimiento) se pudo comprobar que el nivel de significación que relaciona al crecimiento de las plantas con el piso altitudinal y la relación entre crecimiento, piso altitudinal y sustrato; son mayores a un p-valor de 0,05 lo que indica que no existe influencia del piso altitudinal en el crecimiento de las plantas. Por el contrario, el tipo de sustrato al tener un p-valor menor a 0,05 se considera que influye en el efecto que se está analizando, en este caso en el crecimiento de la planta. 
ISSN: 2600-5859

Vol. 4, №2, p. 277-291, abril-junio, 2021

Tabla 5.- Prueba de efectos inter-sujetos. Modelo lineal general, medidas repetidas.

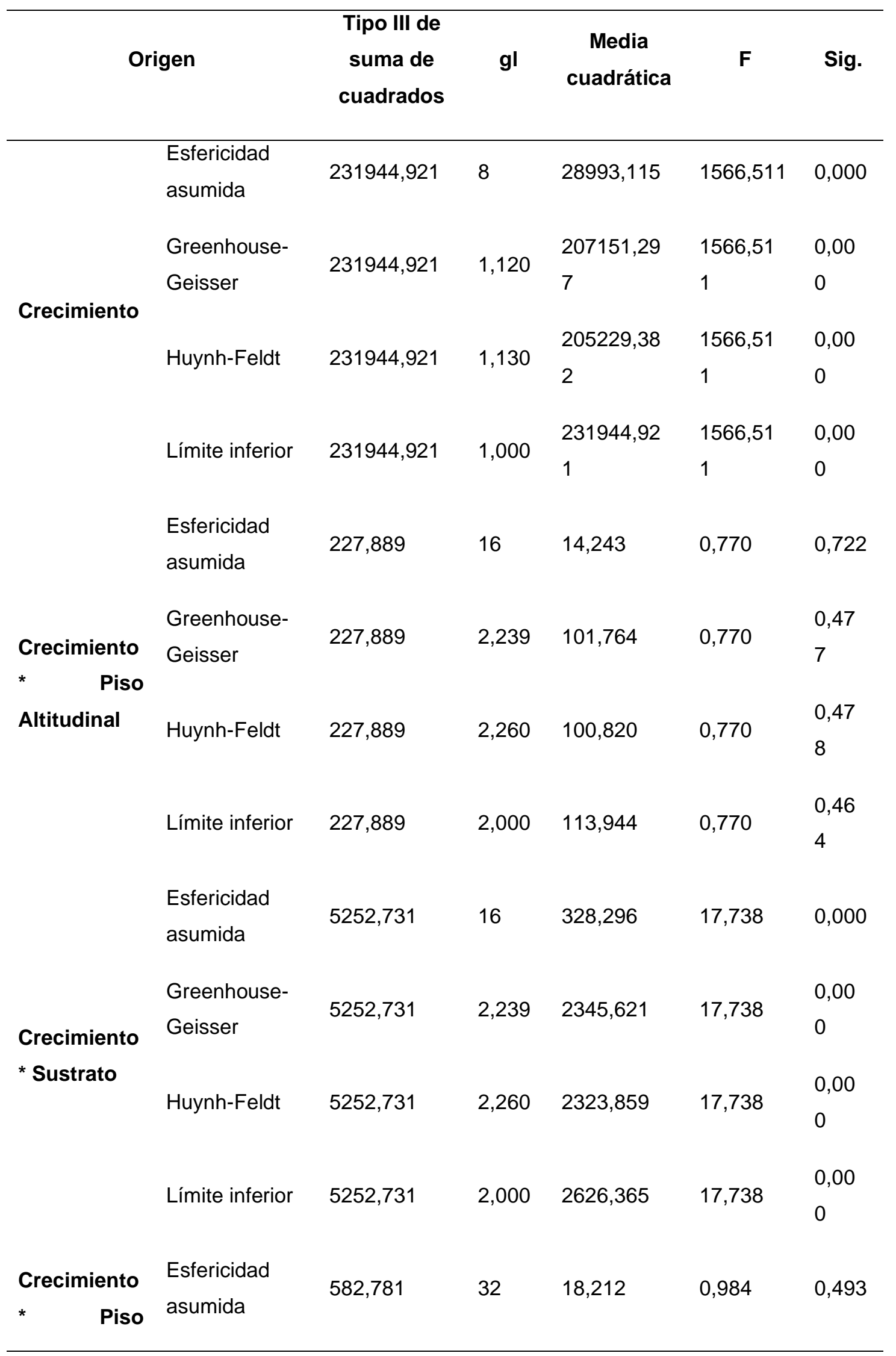


ISSN: 2600-5859

Vol. 4, N², p. 277-291, abril-junio, 2021

\begin{tabular}{|c|c|c|c|c|c|c|}
\hline $\begin{array}{l}\text { Altitudinal * } \\
\text { Sustrato }\end{array}$ & $\begin{array}{l}\text { Greenhouse- } \\
\text { Geisser }\end{array}$ & 582,781 & 4,479 & 130,121 & 0,984 & $\begin{array}{l}0,42 \\
1\end{array}$ \\
\hline & Huynh-Feldt & 582,781 & 4,521 & 128,914 & 0,984 & $\begin{array}{l}0,42 \\
1\end{array}$ \\
\hline & Límite inferior & 582,781 & 4,000 & 145,695 & 0,984 & $\begin{array}{l}0,41 \\
5\end{array}$ \\
\hline & $\begin{array}{l}\text { Esfericidad } \\
\text { asumida }\end{array}$ & 131925,609 & 7128 & 18,508 & & \\
\hline $\begin{array}{l}\text { Error } \\
\text { (Crecimient }\end{array}$ & $\begin{array}{l}\text { Greenhouse- } \\
\text { Geisser }\end{array}$ & 131925,609 & $\begin{array}{l}997,6 \\
42\end{array}$ & 132,237 & & \\
\hline o) & Huynh-Feldt & 131925,609 & $\begin{array}{l}1006, \\
985\end{array}$ & 131,010 & & \\
\hline & Límite inferior & 131926,09 & $\begin{array}{l}891,0 \\
0\end{array}$ & 148,65 & & \\
\hline
\end{tabular}

Fuente: Elaboración propia.

Finalmente, en el análisis de varianza de dos factores (Tabla 6) confirmaron los resultados mencionados anteriormente y se comprueba que existe una influencia del tipo de sustrato sobre el crecimiento de la planta. Mientras que los diferentes pisos altitudinales no influyeron en el crecimiento de la planta.

Tabla 6.- Prueba de efectos inter-sujetos. Modelo lineal general, ANOVA.

\begin{tabular}{lllllll}
\hline Origen & $\begin{array}{l}\text { Tipo III de } \\
\text { suma } \\
\text { cuadrados }\end{array}$ & de & gl & $\begin{array}{l}\text { Media } \\
\text { cuadrática }\end{array}$ & F & Sig. \\
\hline Intersección & 1693685,340 & 1 & 1693685,340 & 2214,033 & 0,000 \\
Piso Altitudinal & 216,814 & 2 & 108,407 & 0,142 & 0,868 \\
Sustrato & 23571,117 & 2 & 11785,559 & 15,406 & 0,000 \\
Piso Altitudinal & $*$ & 134,735 & 4 & 33,684 & 0,044 & 0,996 \\
Sustrato & & & & & & \\
Error & 681595,53 & 891 & 765,88 & &
\end{tabular}

Fuente: Elaboración propia. 


\section{Conclusiones.}

- Dentro de la verificación de la influencia del sustrato y de los diferentes pisos altitudinales se pudo verificar que no existe influencia significativa en la relación entre estos dos factores. Es decir que no importa la combinación de estos factores puesto que el piso altitudinal no altera el crecimiento o sobrevivencia de las especies.

- Se pudo verificar que el tipo de sustrato si influye en referencia al porcentaje de sobrevivientes, esto se vio reflejando ya que al utilizar humus como sustrato el porcentaje se encontró en $78 \%$, al usar turba el porcentaje se situó en $71 \%$ pero al utilizar estiércol de ganado el porcentaje resulto ser del 58\%. La importancia de este valor radica en que usualmente es utilizado como fertilizante o mejorador de la calidad del suelo el estiércol de ganado que se encuentra cercano; esto indicaría que no es recomendable usar el mismo y se debería emplear humus como sustrato para mejorar las oportunidades de la especia para crecer y propagarse.

- El análisis estadístico arrojó los mismos resultados, indicando que existe una diferencia estadísticamente significativa respecto al uso de cada tipo de sustrato frente al crecimiento vertical de la planta (altura), lo que quiere decir que si influye los sustratos en la tasa del crecimiento de la especie. De manera general la tasa de crecimiento se sitúa en $3,01 \mathrm{~cm}$ de alto cada mes y un promedio de crecimiento durante todo el experimento de $24,03 \mathrm{~cm}$.

- Teniendo que el sustrato de humus y turba presentan mayores tasas de crecimiento con 3,01 y $3,02 \mathrm{~cm}$ de crecimiento mensual, respectivamente. El estiércol de ganado produjo un crecimiento de $2,98 \mathrm{~cm}$ mensualmente. Con el sustrato de turba se produjo el mayor crecimiento durante el experimento, dando como resultado un promedio de $24,16 \mathrm{~cm}$.

- Respecto al crecimiento horizontal (diámetro) los resultados fueron similares referente a la altura. De manera general se obtuvo una tasa de crecimiento de 3,45 $\mathrm{mm}$ de diámetro cada mes, con un crecimiento total de 27,58 mm. Sin embargo, contrario a la altura, la utilización de estiércoles y turba presentaron las mayores tasas de crecimiento mensual, $3,50 \mathrm{~mm}$ y $3,48 \mathrm{~mm}$ respectivamente.

- De acuerdo a lo presentado en la figura 1, para el sustrato de estiércol de ganado un promedio de $58 \%$ de sobrevivientes, con un mínimo de $56 \%$ y un máximo de 59\%. Para el caso del sustrato de humus un promedio de $78 \%$ de sobrevivientes, con un mínimo de $76 \%$ y un máximo de $80 \%$. Finalmente, para el sustrato de turba un porcentaje de $71 \%$, con un mínimo de $70 \%$ y un máximo de $71 \%$. Con ello se puede tener una primera aproximación sobre la influencia del sustrato sobre el piso altitudinal. Así mismo, se puede apreciar que el sustrato de humus presentó el mayor rendimiento, seguido de la turba y el estiércol de ganado el que presentó el más bajo rendimiento.

- De acuerdo a los resultados obtenidos se podría indicar que es necesario la utilización de sustratos para mejorar las probabilidades de supervivencia de la especie y es recomendable la utilización de humus, considerando la tasa de individuos vivos, la tasa de crecimiento horizontal y vertical. Cabe indicar que 
para el diseño de un programa de reforestación que sea exitoso es necesario el uso del mejor sustrato posible, con lo cual se suple esta necesidad.

- La replicación del experimento en diferentes pisos altitudinales demostró que puede emplearse la metodología aplicada en este trabajo en otros lugares y obtener resultados similares bajo la utilización de un sustrato específico. Así mismo, tomando en cuenta que es necesario la intervención del ser humano esta no se prolongaría a lo largo del crecimiento de la especie por lo que resulta una técnica económica basada en sustratos orgánicos. Por lo que resulta recomendable la aplicación de la metodología presentada para la propagación de la especie en diferentes pisos altitudinales.

\section{Referencias bibliográficas.}

Acosta, C., Vázquez, N., Villegas, O., Vence, L., \& Acosta, D. (2014). Influencia del vermicompost en el crecimiento de las plantas. Bioagro, 26(2), 107-114.

Ayma, A., \& Padilla, E. (2009). Efecto de la tala de Podocarpus glomeratus (Podocarpaceae) sobre la estructura de un bosque de neblina en los Andes (Cochabamba, Bolivia). Revista Peruana de Biología, 16(1), 73-80.

Díaz, V., \& Núñez, A. (2016). Artículos científicos, tipos de investigación y productividad científica en las Ciencias de la Salud. Rev Cienc Salud, 14(1), 115121.

Global Forest Watch. (2020). Pérdida Mundial de Bosques Primarios. Obtenido de https://www.globalforestwatch.org/dashboards/global/?category=summary\&das hboardPrompts=eyJzaG93UHJvbXB0cyI6dHJ1ZSwicHJvbXB0c1ZpZXdlZCI6 WyJzaGFyZVdpZGdldCIsImRvd25sb2FkRGFzaGJvYXJkU3RhdHMiXSwic2 V0dGluZ3MiOnsic2hvd1Byb21wdHMiOnRydWUsInByb21wdHNWaWV3ZW QiOlsi

Hoehne, L., Altmayer, T., Martini, M., Finatto, J., Brietzke, D., Kuhn, D., . . Cordero, S. (2020). efecto del humus y sustratos del suelo sobre los parámetros de produccion y calidad de las fresas orgánicas. Horticultura Brasileña, 38(1), 1-9.

Keipi, K. (2000). Políticas forestales de América Latina. Washington: Banco Interamericano de Desarrollo.

Moura, L., Santos, S., Almeida, S., Paula, R., Rocha, A., \& Monteiro, A. (2016). Evaluación de sustratos y esporulación de HMA en la producción de plántulas de especies forestales nativas. Revista Árvore, 40(2).

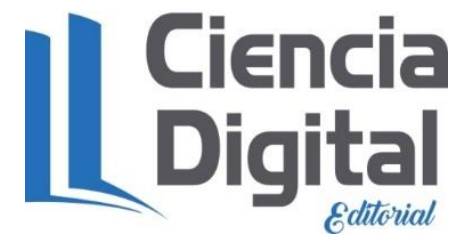




\section{PARA CITAR EL ARTÍCULO INDEXADO.}

Herrera Cabezas, J. E., \& Chim Chi, Y. A. (2021). Implementación de una metodología para la propagación y crecimiento del romerillo. ConcienciaDigital, 4(2), 277-291. https://doi.org/10.33262/concienciadigital.v4i2.1677

\section{¿Ciencia}

El artículo que se publica es de exclusiva responsabilidad de los autores y no necesariamente reflejan el pensamiento de la Revista Conciencia Digital.

El artículo queda en propiedad de la revista y, por tanto, su publicación parcial y/o total en otro medio tiene que ser autorizado por el director de la Revista Conciencia Digital.

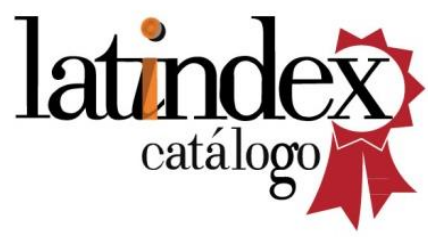

